

\title{
Potent Antimicrobial Agents of 8-Aza-bicyclo[3.2.1]octyl hydrazide Derivatives: Synthesis and Biological Evaluation
}

\section{T. LAKshmi ViveKA ${ }^{1, *}$ and L. Nalanda SharadA ${ }^{2}$}

${ }^{1}$ Department of Chemistry, VNR Vignana Jyothi Institute of Engineering and Technology, Bachupally, Hyderabad-500 090, India ${ }^{2}$ Department of Chemistry, University College of Science, Osmania University, Hyderabad-500 007, India

*Corresponding author: E-mail: lakshmiviveka99@gmail.com

A facile synthesis of 8-aza-bicyclo[3.2.1] octyl hydrazide derivatives 4(a-m) can be achieved by modified Mannich reaction. Novelty of this research involves one pot synthesis of new modified Mannich based triazole derivatives using aromatic aldehydes and active hydrogen containing amides in the presence of ammonia and primary amine. All the synthesized compounds were characterized by IR, ${ }^{1} \mathrm{H}$ NMR,

| ${ }^{13} \mathrm{C}$ NMR and mass spectroscopy. Among all the compounds which are screened for antibacterial and antifungal activities, hydrogen containing thiosemicarbazide moiety and 4,4-diflorocyclohexane carboxylic hydrazide has shown better activity against four bacterial strains and two fungal strains.

| Keywords: Mannich reaction, Triazoles, Hydrazides, Biological activity.

ᄂ - - - - - - - - - - - - - - - - - - - - - - - - - - - - - -

\section{INTRODUCTION}

The protection of carbonyl groups plays an important role in drug design chemistry. The most common method for the preparation of Mannich bases are the reaction of aldehydes with active hydrogen containing amide moieties such as semicarbazide, thiosemicarbazide and 4,4-difluorocyclohexane carboxylic acid hydrazide in the presence of acid or base as catalyst with secondary amine [1-5]. The examples of clinically useful Mannich bases which consist of amino alkyl chain are cocaine, fluoxetine, atropine, ethacrynic acid, procyclidine and so forth.

Mannich bases also known to play a vital role in the development of synthetic pharmaceutical chemistry. Mannich reactions are widely used for the construction of nitrogen containing compounds. These bases have important biological applications such as antifungal [6], antimicrobial [7-13], anticonvulsant $[14,15]$, antitumor [16], antiepileptic activity [17], anti-inflammatory, analgesic [18], antimalarial [19] and antioxidant activity [20].

\section{EXPERIMENTAL}

All the compounds used in the synthesis are of analytical grade. The melting points of the compounds were determined in open head capillary in paraffin bath and are uncorrected. The IR spectra of the compounds were recorded in the region of 4000-400 $\mathrm{cm}^{-1}$ by FT-IR Perkin spectrophotometer. ${ }^{1} \mathrm{H}$ NMR spectra were recorded on Bruker FT NMR $(400 \mathrm{~Hz})$ spectro photometer in DMSO- $d_{6}$. The values of the chemical shift are expressed in $\delta$ ppm as a unit. Mass spectral data were obtained by using a network mass selective detector (Agilent). All the compounds were checked for purity by thin layer chromatography (TLC).

in vitro Antibacterial screening: The compounds (4a-4m) were evaluated for their in vitro antibacterial activity against Escherichia coli (MTCC No. 42), Pseudomonas aeruginosa (MTCC No. 1034), Staphylococcus aureus (MTCC No.3160), Bacillus megaterium (MTCC No. 6544) by disc diffusion method $[21,22]$. It was performed using a LBS agar medium. Each compound and standard were used at a concentration of $100 \mu \mathrm{g} / \mathrm{mL}$ in DMSO. The zone of inhibition was measured after $24 \mathrm{~h}$ incubation at $37^{\circ} \mathrm{C}$.

in vitro Antifungal screening: The compounds $\mathbf{4 a - 4 m}$ were evaluated for their in vitro antifungal activity against Candia albicans (MTCC No: 3017) and Rizopus microsporus var. oligosporus: (MTCC No: 2785) using disc diffusion method with culture media, which provides all essential nutrients for

This is an open access journal, and articles are distributed under the terms of the Creative Commons Attribution-NonCommercial 4.0 International (CC BY-NC 4.0) License, which allows others to copy and redistribute the material in any medium or format, remix, transform, and build upon the material, as long as appropriate credit is given and the new creations are licensed under the identical terms. 
the growth of microorganisms. Potassium dextrose agar (PDA) medium is used for fungal strains. Each compound and standard were used at a concentration of $100 \mu \mathrm{g} / \mathrm{mL}$ in DMSO. The zone of inhibition was measured after $24 \mathrm{~h}$ incubation at $37^{\circ} \mathrm{C}$.

General procedure for the synthesis of $\mathrm{N}$ - $\{[3-(3$-isopropyl5-methyl-[1,2,4]triazol-4-yl)-8-azabicyclo[3.2.1]oct-8-yl]substituted phenyl methyl $\}$ acid hydrazides (4a-m): To an aqueous solution of active hydrogen containing amide, few drops of aqueous ammonia solution (1 eq.) and secondary amine (1 eq.) were added in drops in an ice-cold solution under constant stirring for dissolution. Aromatic aldehydes dissolved in methanol, added dropwise to the above mixture and stirring was continued for $2 \mathrm{~h}$. The formation of compounds were observed within $30 \mathrm{~min}$. Reaction was monitored by TLC, after completion of reaction, the product was filtered and washed with distilled water and dried at $45-50{ }^{\circ} \mathrm{C}$ (Scheme-I).

\section{Spectral data}

$\mathrm{N}^{\prime}$-\{2-Chlorophenyl)-[3-(3-isopropyl-5-methyl-[1,2,4]triazol-4-yl)-8-azabicyclo[3.2.1]oct-8-yl]methyl\}hydrazine carboxamide (4a): White solid, yield: $75.85 \%$, m.p. 230$232{ }^{\circ} \mathrm{C}$. IR (KBr, $\left.v_{\max }, \mathrm{cm}^{-1}\right): 3464,3242,3151,3060,2987$, 1722, 1654, 1591, 1510, 1415, 1344, 1278, 1219, 1157, 1093, $1051,1033,918,852,736,627 .{ }^{1} \mathrm{H} \mathrm{NMR}$ (DMSO- $\left.d_{6}, 400 \mathrm{MHz}\right)$ : $\delta 10.49$ (s, $2 \mathrm{H},-\mathrm{NH}_{2}, \mathrm{D}_{2} \mathrm{O}$ exchangeable), 8.26 (s, $1 \mathrm{H}$, chiral proton), 8.15-8.18 (m, $\left.1 \mathrm{H}, \mathrm{Ar}-\mathrm{H}, J_{1}=6.023 \mathrm{~Hz}, J_{2}=3.51 \mathrm{~Hz}\right)$, 7.47-7.49 (m, 1H, Ar-H, $J_{1}=5.27 \mathrm{~Hz}, J_{2}=3.26 \mathrm{~Hz}, J=7.027$ $\mathrm{Hz}), 7.368-7.397\left(\mathrm{~m}, 2 \mathrm{H}, \mathrm{Ar}-\mathrm{H}, J_{2}=3.514 \mathrm{~Hz}, J_{2}=6.023 \mathrm{~Hz}\right)$, 6.589 (br, s, 2H, -2NH), $3.789\left(\mathrm{~s}, 3 \mathrm{H},-\mathrm{CH}_{3}\right), 3.46\left(\mathrm{~m}, 1 \mathrm{H}, \mathrm{H}_{\mathrm{a}}\right)$, $3.31-3.36(1 \mathrm{H}, \mathrm{m}, J=9.53 \mathrm{~Hz}), 2.668-2.677(\mathrm{~m}, 2 \mathrm{H}, J=1.757$ $\mathrm{Hz}), 2.326-2.335(\mathrm{~m}, 2 \mathrm{H}, J=1.757 \mathrm{~Hz}), 1.88-1.97\left(\mathrm{~m}, 4 \mathrm{H}, J_{1}\right.$ $\left.=1.225 \mathrm{~Hz}, J_{2}=2.008 \mathrm{~Hz}\right), 1.253-1.236\left(\mathrm{~d}, 6 \mathrm{H}, 2 \times \mathrm{CH}_{3}, J=\right.$ $7.027 \mathrm{~Hz}) ;{ }^{13} \mathrm{C}$ NMR: $8.1\left(\mathrm{CH}_{3}\right), 24.4\left(2 \times \mathrm{CH}_{3}\right), 28.4(2 \times$ $\left.\mathrm{CH}_{2}\right), 26.3$ (1CH)-isopropyl), $31.8(1 \mathrm{CH}), 36.6\left(2 \times \mathrm{CH}_{2}\right), 52.7$ $(2 \times \mathrm{CH}), 65.2(1 \mathrm{CH}$, adjacent to arom. ring $), 126.3,128.4$, 128.6, 130.4, 136.7, 137.3 (6C-arom. ring), $158.8(-\mathrm{C}=\mathrm{O}$, amide), 163.7 (2C, imine $(\mathrm{C}=\mathrm{N}))$. Mass: $m / z$ : $429(\mathrm{M}-2), 391$ ((M-2)-isopropyl), 414 ((M-2)-NH $\mathrm{NH}_{3}, 301,309,279$.

$\mathrm{N}^{\prime}$-\{4-Propane-2-yl-phenyl)-[3-(3-isopropyl-5-methyl[1,2,4]triazol-4-yl)-8-azabicyclo[3.2.1]oct-8-yl]methyl\}hydrazine carboxamide (4b): White solid, yield: $79.82 \%$, m.p. $215-217^{\circ} \mathrm{C}$. IR $\left(\mathrm{KBr}, v_{\max }, \mathrm{cm}^{-1}\right): 3458,3344,3068,2951$, 2864, 1683, 1643, 1597, 1508, 1438, 1357, 1286, 1234, 1134, 1087, 958, 933, 829, 754. ${ }^{1} \mathrm{H}$ NMR (DMSO- $d_{6}, 400$ $\mathrm{MHz}): \delta 10.18(\mathrm{~s}, 1 \mathrm{H},-\mathrm{NH}), 7.804(\mathrm{~s}, 1 \mathrm{H}$, -chiral proton) 7.6327.611 (d, 2H, Ar-H, $J=8.282 \mathrm{~Hz}$ ), 7.24-7.261 (d, 2H, Ar-H, $J$ $=8.282 \mathrm{~Hz}), 6.451\left(\mathrm{~s}, 2 \mathrm{H},-\mathrm{NH}_{2}, \mathrm{D}_{2} \mathrm{O}\right.$-exchangeable $), 4.236$ (s,1H,-NH-), 3.991-4.017 (m, 2H, $J_{1}=5.52 \mathrm{~Hz}, J_{2}=4.768$ $\mathrm{Hz}), 3.779-3.805\left(\mathrm{~m}, 1 \mathrm{H}, \mathrm{H}_{\mathrm{a}}, J_{1}=5.52 \mathrm{~Hz}, J_{2}=4.517 \mathrm{~Hz}\right)$, $3.338\left(\mathrm{~s}, 3 \mathrm{H},-\mathrm{CH}_{3}\right), 2.86-2.929\left(\mathrm{~m}, 6 \mathrm{H}, J_{1}=6.776 \mathrm{~Hz}, J_{2}=\right.$ $7.027 \mathrm{~Hz}), 2.669-2.677(\mathrm{~m}, 2 \mathrm{H}, J=1.757 \mathrm{~Hz}), 2.326-2.336$ $\left(\mathrm{m}, 2 \mathrm{H}, J_{1}=1.757 \mathrm{~Hz}, J_{2}=2.008 \mathrm{~Hz}\right), 1.193-1.210(\mathrm{~d}, 12 \mathrm{H}, J$ $=6.776 \mathrm{~Hz}) ;{ }^{13} \mathrm{C} \mathrm{NMR}: \delta 8.1\left(\mathrm{CH}_{3}\right), 24.4\left(4 \times \mathrm{CH}_{3}\right), 28.4(2 \times$ $\left.\mathrm{CH}_{2}\right), 26.3(1 \mathrm{CH})$-isopropyl), $31.8(1 \mathrm{CH}), 31.7(1 \mathrm{CH}), 36.6$ $\left(2 \times \mathrm{CH}_{2}\right), 52.7(2 \times \mathrm{CH}), 74.3(1 \mathrm{CH}$, adjacent to arom. ring), 126, 128.7, 128.6, 133.4, 147.2 (4C-arom. ring), $158.8(\mathrm{C}=\mathrm{O}$, amide), $163.7(2 \mathrm{C}$, imine $(\mathrm{C}=\mathrm{N}))$. Mass $(\mathrm{m} / z): 412\left(\mathrm{M}-\mathrm{N}_{2} \mathrm{H}_{2}\right)$, 427, 434, $441(\mathrm{M}+2)$.
$\mathrm{N}^{\prime}$-\{4-Propane-2-yl-phenyl)-[3-(3-isopropyl-5-methyl[1,2,4]triazol-4-yl)-8-azabicyclo[3.2.1]oct-8-yl]methyl\}hydrazine carbothiamide (4c): White solid, yield: $67.27 \%$, m.p. $150-152^{\circ} \mathrm{C}$. IR $\left(\mathrm{KBr}, v_{\max }, \mathrm{cm}^{-1}\right): 3414,3277,3153,3138$, 2972, 2954, 1587, 1531, 1469, 1365, 1280, 1228, 1180, 1141, 1078, 1053, 1014, 954, 931, 873, 821, 711. ' H NMR (DMSO$d_{6}, 400 \mathrm{MHz}$ ): $\delta 11.38\left(\mathrm{~s}, 2 \mathrm{H},-\mathrm{NH}_{2}\right.$ ), 8.178 (s, 1H, NH), 7.944 (s, 1H, NH), $\mathrm{D}_{2} \mathrm{O}$-exchangeable protons, $8.034(\mathrm{~s}, 1 \mathrm{H}$, chiral proton), 7.70-7.72 (d, 2H, Ar-H, $J=8.031 \mathrm{~Hz}) 7.284-7.305$ (d, 2H, Ar-H, $J=8.282 \mathrm{~Hz}), 2.664-2.682\left(\mathrm{~m}, 2 \mathrm{H}, J_{1}=1.757\right.$ $\left.\mathrm{Hz}, J_{2}=2.008 \mathrm{~Hz}\right), 2.326-2.336\left(\mathrm{~m}, 2 \mathrm{H}, J_{1}=1.757 \mathrm{~Hz}, J_{2}=\right.$ $2.008 \mathrm{~Hz},-\mathrm{CH}\left(\mathrm{CH}_{3}\right)_{2}, 2.863-2.963\left(\mathrm{~m}, 4 \mathrm{H}, J_{1}=6.776 \mathrm{~Hz}, J_{2}=\right.$ $13.803 \mathrm{~Hz}$ ), $3.36\left(\mathrm{~m}, 1 \mathrm{H}, \mathrm{H}_{\mathrm{a}}\right), 3.78$ (s, 3H, $\left.-\mathrm{CH}_{3}\right), 1.199-1.232$ (d, $\left.12 \mathrm{H}, 4 \times \mathrm{CH}_{3}, J=6.776 \mathrm{~Hz}\right), 1.422-1.51(\mathrm{~m}, 4 \mathrm{H}) .{ }^{13} \mathrm{C} \mathrm{NMR}$ :

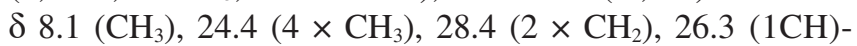
isopropyl), $31.8(1 \mathrm{CH}), 31.7(1 \mathrm{CH}), 36.6\left(2 \times \mathrm{CH}_{2}\right), 52.7(2 \times$ $\mathrm{CH}), 75$ (1CH, adjacent to arom. ring), 126, 128.7, 128.6, 133.4, 147.2 (4C-arom. ring), 163.7 (2C, imine $(\mathrm{C}=\mathrm{N})), 186$ $(-\mathrm{C}=\mathrm{S})$. Mass $(\mathrm{m} / \mathrm{z}): 456.2(\mathrm{M}+1), 439\left(\mathrm{M}-\mathrm{NH}_{2}\right), 412(\mathrm{M}-$ isopropyl), $395.29\left(\mathrm{M}-\mathrm{CSNH}_{2}\right), 365\left(\mathrm{M}-\mathrm{CSN}_{3} \mathrm{H}_{3}\right)$.

$\mathrm{N}^{\prime}$-\{(2E)-3-phenyl-prop-2-en-1-yl)-[3-(3-isopropyl-5methyl-[1,2,4]triazol-4-yl)-8-azabicyclo[3.2.1]oct-8-yl]methyl $\}$ hydrazine carbothiamide (4d): Pale brown solid, yield: $82.21 \%$, m.p. $208-210^{\circ} \mathrm{C}$. IR $\left(\mathrm{KBr}, v_{\max }, \mathrm{cm}^{-1}\right)$ : 3456 , 3344, 3140, 3080, 2980, 1710, 1670, 1645, 1589, 1512, 1421, 1298, 1253, 1178, 1139, 974, 740, 684. ${ }^{1} \mathrm{H}$ NMR (DMSO-d $400 \mathrm{MHz}): \delta 10.207$ (s, 2H, $\mathrm{NH}_{2}$ ), 7.674-7.694 (d, 1H, chiral proton, $J=8.031 \mathrm{~Hz}$ ), 7.519- $7.537(\mathrm{~d}, 2 \mathrm{H}, \mathrm{Ar}-\mathrm{H}, J=7.278$ $\mathrm{Hz}$ ), 7.352-7.389 (t, 2H, Ar-H, J=7.278 Hz, 7.529 Hz), 7.7247.310 (t, 1H, ArH, $J=7.278$ Hz), 6.864-6.88 (d, 2H, CH=CH, $J=6.274 \mathrm{~Hz}), 6.294(\mathrm{~s}, 2 \mathrm{H}, 2 \times \mathrm{NH}), 3.783-3.723(\mathrm{~m}, 1 \mathrm{H}$, $\left.\mathrm{CH}\left(\mathrm{CH}_{3}\right)_{2}\right), 3.438-3.471\left(\mathrm{~m}, 1 \mathrm{H}, \mathrm{H}_{\mathrm{a}}, J=6.776 \mathrm{~Hz}\right), 3.32(\mathrm{~s}$, $\left.3 \mathrm{H},-\mathrm{CH}_{3}\right), 2.672-2.676(\mathrm{~m}, 2 \mathrm{H}, J=1.757 \mathrm{~Hz}), 2.325-2.334$ $(\mathrm{m}, J=1.757 \mathrm{~Hz}), 1.728-1.742\left(\mathrm{~m}, 2 \mathrm{H}, J_{1}=2.008 \mathrm{~Hz}, J_{2}=\right.$ $1.25 \mathrm{~Hz}), 1.534-1.537(\mathrm{~m}, 2 \mathrm{H}, J=1.25 \mathrm{~Hz}),{ }^{13} \mathrm{C} \mathrm{NMR}: \delta 8.1$ $\left(\mathrm{CH}_{3}\right), 24.4\left(2 \times \mathrm{CH}_{3}\right), 28.5\left(2 \times \mathrm{CH}_{2}\right), 26.3(1 \mathrm{CH})$-isopropyl), $31.8(1 \mathrm{CH}), 36.7\left(2 \times \mathrm{CH}_{2}\right), 53.2(2 \times \mathrm{CH}), 74.4(1 \mathrm{CH}$, adjacent to arom. ring), 127.4, 123.3 (2C, vinyl group), 126.2, 127.7, 128.4, 134.9, (4C-arom. ring), 163.7 (2C, imine $(\mathrm{C}=\mathrm{N})), 186$ $(-\mathrm{C}=\mathrm{S})$. Mass $(\mathrm{m} / \mathrm{z}): 440.25(\mathrm{M}+1), 396.20$ (M-isopropyl), $379.52\left(\mathrm{M}-\mathrm{CSNH}_{2}\right)$.

$\mathbf{N}^{\prime}$-\{(2-Chloro-quinolin-3-yl)-[3,3-isopropyl-5-methyl)[1,2,4]triazol-4-yl)-8-azabicyclo[3.2.1] oct-8-yl]-(3-nitrophenyl)methyl]hydrazine carbothiamide (4e): Yellow solid, yield: $75.0 \%$ m.p. $222-225^{\circ} \mathrm{C}$. IR $\left(\mathrm{KBr}, v_{\max }, \mathrm{cm}^{-1}\right)$ : 3460 , 3342, 3143, 3072, 2980, 1651, 1597, 1525, 1490, 1448, 1352, 1274, 1211, 1184, 1128, 1105, 1045, 960, 931, 844, 813, 758, 686. ${ }^{1} \mathrm{H}$ NMR (DMSO- $d_{6}, 400 \mathrm{MHz}$ ): $\delta 12.063$ (s, $\left.1 \mathrm{H},-\mathrm{NH}\right)$, 11.83 (s, 2H, $\left.-\mathrm{NH}_{2}\right), 11.654(\mathrm{~s}, 1 \mathrm{H},-\mathrm{NH}) \mathrm{D}_{2} \mathrm{O}$-exchangeable protons, 8.52-8.50 (d, $1 \mathrm{H}$, chiral proton), 8.023-7.959 (dd, $2 \mathrm{H}$, $\left.J_{1}=8.03 \mathrm{~Hz}, \mathrm{~J}_{2}=8.28 \mathrm{~Hz}\right), 7.84-7.87(\mathrm{t}, 1 \mathrm{H}, \mathrm{Ar}-\mathrm{H}, J=7.278$ $\mathrm{Hz}), 7.732-7.69$ (t, 1H, Ar-H, $J=7.27 \mathrm{~Hz}$ ),4.136-4.175 (m, $\left.1 \mathrm{H}, \mathrm{H}_{\mathrm{a}}, J=5.27 \mathrm{~Hz}\right), 3.48\left(\mathrm{~s}, 3 \mathrm{H},-\mathrm{CH}_{3}\right), 3.28(\mathrm{~m}, 1 \mathrm{H}, J=5.27$ $\mathrm{Hz}), 2.353$ (m, 2H), 1.868(m, 4H), 1.446 (m, 4H), 1.25-1.26 $\left(\mathrm{d}, 6 \mathrm{H}, J=6.77 \mathrm{~Hz}, 2 \times \mathrm{CH}_{3}\right) .{ }^{13} \mathrm{C} \mathrm{NMR}: \delta 8.1\left(1 \mathrm{C}, \mathrm{CH}_{3}\right), 24.4$ $\left(2 \times \mathrm{CH}_{3}\right), 28.4\left(2 \times \mathrm{CH}_{2}\right), 26.3(1 \mathrm{CH})$-isopropyl), $31.8(1 \mathrm{CH})$, $36.6\left(2 \times \mathrm{CH}_{2}\right), 52.7(2 \times \mathrm{CH}), 66.3(1 \mathrm{CH}$, adjacent to arom. ring), 126, 126.3, 126.8, 127.6, 129.2, 130.9, 135.9, 146.8, 
<smiles>Cc1nnc(C(C)C)n1C1CC2CCCC(C1)N2</smiles>

(1)<smiles>Cc1nnc(C(C)C)n1C1CC2CCCC(C1)N2</smiles>

(1)<smiles>NNC(=O)C1CCC(F)(F)CC1</smiles>

(3c)<smiles>C1CC2CCC1C2</smiles>

$3(a-b)$<smiles>[X]C(N)NN</smiles>
Methanol and ammonia solution<smiles>[X]C(N)NN</smiles><smiles>[R]c1cccc(C(C)N2C3CCC2CC(n2c(C)nnc2C(C)C)C3)c1</smiles>

$\overrightarrow{\text { Methanol and ammonia solution }}$

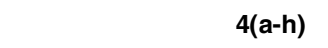

4(a-h)

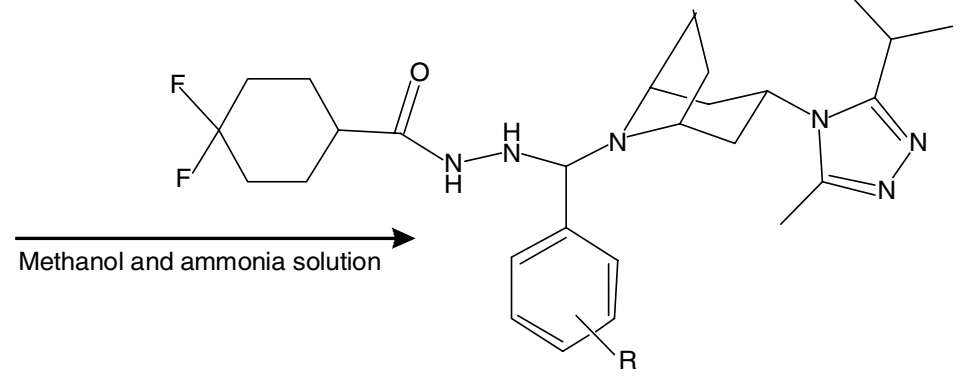

4(i-m)<smiles>Cc1nnc(C(C)C)n1C1CC2CCC1N2C(/C=C/c1ccccc1)NNC(N)=S</smiles><smiles>COc1ccc(C(NNC(N)=S)N2C3CCC(C3)N2C(NNC(N)=S)c2ccc(O[Ga])cc2)cc1</smiles><smiles>COc1cc(C(NNC(=O)C2CCC(F)(F)CC2)N2C3CCC2C32CC3CC32n2c(C)nnc2C(C)C)cc(OC)c1OC</smiles>
$4 i$

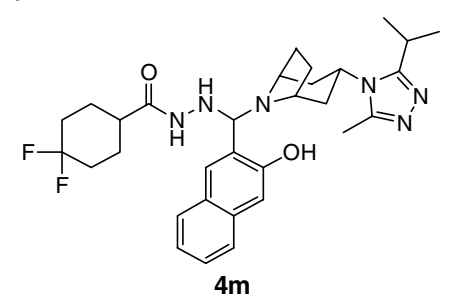

Scheme-I: Synthetic route of compounds $4(\mathbf{a}-\mathbf{m})$ 
157.7 (9C-quinoline ring)), $163.7(2 \mathrm{C}$, imine $(\mathrm{C}=\mathrm{N})), 186(-$ $\mathrm{C}=\mathrm{S})$. Mass $(\mathrm{m} / \mathrm{z}): 499,461.8(\mathrm{M}-\mathrm{Cl}), 437.8\left(\mathrm{M}-\mathrm{CSNH}_{2}\right)$, 279.2.

$\mathrm{N}^{\prime}$-\{(3-Nitro phenyl)-[3-(3-isopropyl-5-methyl[1,2,4]triazol-4-yl)-8-azabicyclo[3.2.1]oct-8-yl]methyl\}hydrazine carbothiamide (4f): Cream solid, yield: 69.0\%, m.p. 232-234 ${ }^{\circ} \mathrm{C}$. IR $\left(\mathrm{KBr}, v_{\max }, \mathrm{cm}^{-1}\right)$ : 3392, 3226, 3153, 3030, 2980, 1600, 1525,1471, 1346, 1294, 1226, 1163, 1105, 1068, 937, 887, 842, $815,732,705,673 .{ }^{1} \mathrm{H}$ NMR (DMSO- $\left.d_{6}, 400 \mathrm{MHz}\right): \delta 11.704$ (s, $1 \mathrm{H},-\mathrm{NH}), 11.513(\mathrm{~s}, 1 \mathrm{H},-\mathrm{NH}) \mathrm{D}_{2} \mathrm{O}$-exchangeable protons, 8.518-8.509 (t, $\left.1 \mathrm{H}, \mathrm{Ar}-\mathrm{H}, J_{1}=1.757 \mathrm{~Hz}, J_{2}=2.008 \mathrm{~Hz}\right), 8.457$ $8.447\left(\mathrm{t}, 1 \mathrm{H}, \mathrm{Ar}-\mathrm{H}, J_{1}=1.757 \mathrm{~Hz}, J_{2}=2.008 \mathrm{~Hz}\right), 8.16-8.18$ $(\mathrm{d}, 1 \mathrm{H}, \mathrm{Ar}-\mathrm{H}, J=7.78 \mathrm{~Hz}), 8.311-8.32(\mathrm{~d}, 1 \mathrm{H}$, chiral proton $J$ $=3.514 \mathrm{~Hz}), 7.706-7.755\left(\mathrm{dt}, 1 \mathrm{H}, \mathrm{Ar}-\mathrm{H}, J_{1}=8.031 \mathrm{~Hz}, J_{2}=4.015\right.$ $\left.\mathrm{Hz}, J_{3}=7.780 \mathrm{~Hz}\right) 4.084-4.124\left(\mathrm{~m}, 1 \mathrm{H},-\mathrm{CH}\left(\mathrm{CH}_{3}\right)_{2}\right), 3.334(\mathrm{~s}$, $\left.3 \mathrm{H}, \mathrm{CH}_{3}\right), 3.67\left(\mathrm{~m}, 1 \mathrm{H}, \mathrm{H}_{\mathrm{a}}, J=5.270 \mathrm{~Hz}\right), 2.408(\mathrm{~m}, 2 \mathrm{H}, J=$ $10.79 \mathrm{~Hz}), 1.602-2.110(\mathrm{~m}, 8 \mathrm{H}, J=4.015 \mathrm{~Hz}, 1.234-1.250$ (d, $\left.6 \mathrm{H},-2 \times \mathrm{CH}_{3}, J=6.776 \mathrm{~Hz}\right) .{ }^{13} \mathrm{C}$ NMR: $\delta 8.1\left(\mathrm{CH}_{3}\right), 24.4(2 \times$ $\left.\mathrm{CH}_{3}\right), 28.4\left(2 \times \mathrm{CH}_{2}\right), 26.3(1 \mathrm{CH})$-isopropyl), $31.8(1 \mathrm{CH}), 36.6$ $\left(2 \times \mathrm{CH}_{2}\right), 52.7(2 \times \mathrm{CH}), 74(1 \mathrm{CH}$, adjacent to arom. ring), 122.1, 124.1, 129.1, 135.1, 137.2, 148.1 (6C-arom. ring), 163.7 $(2 \mathrm{C}$, imine $(\mathrm{C}=\mathrm{N})), 186(-\mathrm{C}=\mathrm{S})$. Mass $(\mathrm{m} / \mathrm{z}): 459.22(\mathrm{M}+1)$, 415.49 (M-isopropyl), $414.8\left[(\mathrm{M}+2)-\mathrm{NO}_{2}\right], 398.25\left(\mathrm{M}-\mathrm{CSNH}_{2}\right)$.

$\mathbf{N}^{\prime}$-\{(3-Methoxyphenyl)-[3-(3-isopropyl-5-methyl$[1,2,4]$ triazol-4-yl)-8-azabicyclo[3.2.1]oct-8-yl]methyl\}hydrazine carbothiamide (4g): White shiny solid, yield: $79.65 \%$, m.p. $158-160^{\circ} \mathrm{C}$. IR (KBr, $\left.v_{\max }, \mathrm{cm}^{-1}\right): 3392,3157$, 3034, 2980, 1595, 1541, 1508, 1465, 1444, 1415, 1377, 1294 , $1249,1161,1101,1051,1024,952,927,883,819,690 .{ }^{1} \mathrm{H}$ NMR (DMSO- $d_{6}, 400 \mathrm{MHz}$ ): $\delta 10.2(\mathrm{~s}, 1 \mathrm{H},-\mathrm{NH}), 7.804$ (s, $1 \mathrm{H}$, -chiral proton), 7.632-7.611 (d, 2H, Ar- $\mathrm{H}, J=8.282 \mathrm{~Hz}$ ), 7.24-7.26 (d, $2 \mathrm{H}, \mathrm{Ar}-\mathrm{H}, J=8.282 \mathrm{~Hz}), 6.451$ (s, $2 \mathrm{H},-\mathrm{NH}_{2}$, D2O-exchangeable), 4.36 (s,1H, -NH-), 3.991-4.017 (m, 1H, $\left.J_{1}=5.27 \mathrm{~Hz}, J_{2}=4.768 \mathrm{~Hz}\right), 3.779-3.805\left(\mathrm{~m}, 1 \mathrm{H}, \mathrm{H}_{\mathrm{a}}, J_{1}=\right.$ $\left.5.52 \mathrm{~Hz}, J_{2}=4.517 \mathrm{~Hz}\right), 3.75\left(\mathrm{~s}, 3 \mathrm{H},-\mathrm{OCH}_{3}\right), 3.34(\mathrm{~s}, 3 \mathrm{H}$, $\left.\mathrm{CH}_{3}\right), 2.86-2.929\left(\mathrm{~m}, 6 \mathrm{H}, J_{1}=6.776 \mathrm{~Hz}, J_{2}=7.027 \mathrm{~Hz}\right), 2.669$ $2.677\left(\mathrm{~m}, 2 \mathrm{H}, J=1.757 \mathrm{~Hz}, 2.326-2.336\left(\mathrm{~m}, 2 \mathrm{H}, J_{1}=1.757\right.\right.$ $\left.\mathrm{Hz}, J_{2}=2.008 \mathrm{~Hz}\right), 1.193-1.210(\mathrm{~d}, 6 \mathrm{H}, J=6.776 \mathrm{~Hz}) .{ }^{13} \mathrm{C} \mathrm{NMR}$ : $\delta 8.1\left(\mathrm{CH}_{3}\right), 24.4\left(2 \times \mathrm{CH}_{3}\right), 28.4\left(2 \times \mathrm{CH}_{2}\right), 26.3(1 \mathrm{CH})$ isopropyl), $31.8(1 \mathrm{CH}), 36.6\left(2 \times \mathrm{CH}_{2}\right), 52.7(2 \times \mathrm{CH}), 56(1 \mathrm{C}$, $\left.\mathrm{OCH}_{3}\right), 75(1 \mathrm{CH}$, adjacent to arom. ring), 113.8, 128.6, 130, 160.5 (4C-arom. ring), $163.7(2 \mathrm{C}$, imine $(\mathrm{C}=\mathrm{N})), 186(-\mathrm{C}=\mathrm{S})$. Mass $(\mathrm{m} / \mathrm{z}): 441.25(\mathrm{M}+1), 413.58\left(\mathrm{M}-\mathrm{OCH}_{3}\right), 383.6\left(\mathrm{M}-\mathrm{CSNH}_{2}\right)$.

$\mathbf{N}^{\prime}$-\{(2,4-Dimethoxy-phenyl)-[3-(3-isopropyl-5-methyl[1,2,4] triazol-4-yl)-8-azabicyclo[3.2.1]oct-8-yl]methyl\}hydrazine carbothiamide (4h): Pale yellow solid, yield: $78.24 \%$, m.p. $193-195^{\circ} \mathrm{C}$. IR (KBr, $\left.v_{\max }, \mathrm{cm}^{-1}\right)$ : 3448, 3340, 3257, 3120, 2972, 1685, 1589, 1527, 1504, 1450, 1421, 1365, 1319, 1269, 1207, 1166, 1109, 1024, 939, 813, 738. ${ }^{1} \mathrm{H}$ NMR (DMSO- $\left.d_{6}, 400 \mathrm{MHz}\right): \delta 10.53(\mathrm{~s}, 2 \mathrm{H}, 2 \times \mathrm{NH}), 8.665(\mathrm{~s}, 2 \mathrm{H}$, -NH-), 8.065 (s, 1H, chiral proton), 7.77-7.78 (s, Ar-H, 1H, $J=$ $8.69 \mathrm{~Hz}), 6.54-6.56(\mathrm{dd}, 1 \mathrm{H}, J=6.4 \mathrm{~Hz}), 6.452-6.448(\mathrm{~d}, 1 \mathrm{H}$, $J=2.136 \mathrm{~Hz}), 3.85\left(\mathrm{~s}, 6 \mathrm{H},-\mathrm{OCH}_{3}\right), 3.73(\mathrm{~m}, 1 \mathrm{H}, J=5.27 \mathrm{~Hz})$, 3.23-3.28 (m, 1H, - $\left.\mathrm{CH}\left(\mathrm{CH}_{3}\right)_{2}\right), 2.74\left(\mathrm{~s}, 3 \mathrm{H},-\mathrm{CH}_{3}\right), 2.35$ (m, $2 \mathrm{H}), 1.59-2.17(\mathrm{~m}, 8 \mathrm{H}), 1.29\left(\mathrm{~d}, 6 \mathrm{H},-2 \times \mathrm{CH}_{3}, J=6.77 \mathrm{~Hz}\right) .{ }^{13} \mathrm{C}$ NMR: $\delta 8.1\left(\mathrm{CH}_{3}\right), 24.4\left(2 \times \mathrm{CH}_{3}\right), 28.4\left(2 \times \mathrm{CH}_{2}\right), 26.3(1 \mathrm{CH})-$ isopropyl), $31.8(1 \mathrm{CH}), 36.6\left(2 \times \mathrm{CH}_{2}\right), 52.7(2 \times \mathrm{C} \mathrm{H}), 56(2 \mathrm{C}$, $\left.2 \times \mathrm{OCH}_{3}\right), 65.1(1 \mathrm{CH}$, adjacent to arom. ring), 99.4, 106.1,
161.5, 163.5 (4C-arom. ring), $163.7(2 \mathrm{C}$, imine $(\mathrm{C}=\mathrm{N})), 186$ $(-\mathrm{C}=\mathrm{S})$. Mass $(\mathrm{m} / \mathrm{z})$ : $474.26(\mathrm{M}+1), 431.5$ (M-isopropyl), $443.61\left(\mathrm{M}-\mathrm{OCH}_{3}\right), 441.65\left(\mathrm{M}-\mathrm{N}_{2} \mathrm{H}_{2}\right)$.

4,4-Difluoro-cyclohexanecarboxylic acid $N^{\prime}$-\{[3-3-isopropyl-5-methyl)[1,2,4]triazol-4-yl)-8-azabicyclo[3.2.1]oct-8-yl]-(3-nitro phenyl)methyl]hydrazide (4i): Off white solid, yield: $76.34 \%$, m.p. $187-189^{\circ} \mathrm{C}$. IR $\left(\mathrm{KBr}, v_{\max }, \mathrm{cm}^{-1}\right)$ : 3456, 3346, 3217, 3066, 2974, 2864, 1670, 1595, 1521, 1408, 1348, 1232, 1178, 1141, 1103, 968, 904, 813, 738, 678. ${ }^{1} \mathrm{H} N \mathrm{MR}$ (DMSO-d $\left.d_{6}, 400 \mathrm{MHz}\right): \delta 11.704$ (s, $\left.1 \mathrm{H},-\mathrm{NH}\right), 11.513$ (s, $1 \mathrm{H}$, $\mathrm{NH}) \mathrm{D}_{2} \mathrm{O}$-exchangeable protons, 8.518-8.509 (t, $1 \mathrm{H}, \mathrm{Ar}-\mathrm{H}, J_{1}$ $\left.=1.757 \mathrm{~Hz}, J_{2}=2.008 \mathrm{~Hz}\right), 8.457-8.447\left(\mathrm{t}, 1 \mathrm{H}, \mathrm{Ar}-\mathrm{H}, J_{1}=\right.$ $\left.1.757 \mathrm{~Hz}, J_{2}=2.008 \mathrm{~Hz}\right), 8.16-8.18(\mathrm{~d}, 1 \mathrm{H}, \mathrm{Ar}-\mathrm{H}, J=7.78 \mathrm{~Hz})$, 8.31-8.32 (d, $1 \mathrm{H}$, chiral proton $J=3.514 \mathrm{~Hz}), 7.706-7.755$ $\left(\mathrm{dt}, 1 \mathrm{H}, \mathrm{Ar}-\mathrm{H}, J_{1}=8.031 \mathrm{~Hz}, J_{2}=4.015 \mathrm{~Hz}, J_{3}=7.780 \mathrm{~Hz}\right.$ ), 4.084-4.124 (m, $\left.1 \mathrm{H},-\mathrm{CH}\left(\mathrm{CH}_{3}\right)_{2}\right), 3.334\left(\mathrm{~s}, 3 \mathrm{H}, \mathrm{CH}_{3}\right), 3.162-$ $3.175\left(\mathrm{~d}, 1 \mathrm{H}, \mathrm{H}_{\mathrm{a}}, J=5.270 \mathrm{~Hz}\right), 2.408(\mathrm{~m}, 2 \mathrm{H}, J=10.79 \mathrm{~Hz})$, $2.335-2.326\left(\mathrm{~m}, 1 \mathrm{H}, J_{l}=1.757 \mathrm{~Hz}, J_{2}=2.008 \mathrm{~Hz}\right.$, cyclohexyl, $\mathrm{CH}-), 1.602-2.110\left(\mathrm{~m}, 16 \mathrm{H}, J=13.803 \mathrm{~Hz}, J_{2}=4.015 \mathrm{~Hz}, J_{3}\right.$ $=13.30 \mathrm{~Hz}), 1.234-1.250\left(\mathrm{~d}, 6 \mathrm{H},-2 \times \mathrm{CH}_{3}, J=6.776 \mathrm{~Hz}\right) .{ }^{13} \mathrm{C}$ NMR: $\delta 8.1\left(\mathrm{CH}_{3}\right), 14.2\left(2 \times \mathrm{CH}_{2}\right), 24.4\left(2 \times \mathrm{CH}_{3}\right), 28.4\left(2 \times \mathrm{CH}_{2}\right)$, $26.3(1 \mathrm{CH})$-isopropyl), $31.8(1 \mathrm{CH}), 34.4\left(2 \times \mathrm{CH}_{2}\right), 36.6(2 \times$ $\left.\mathrm{CH}_{2}\right), 42$ (1CH-difluoro cyclohexyl ring), $52.7(2 \times \mathrm{CH}), 73.8$ (1CH, adjacent to arom. ring), 108.3 (1C-geminal fluoro carbon), 122.1, 124.1, 129.1, 135.1, 137.2, 148.1 (6C-arom. ring), 163.7 (2C, imine $(\mathrm{C}=\mathrm{N}), 177.9(-\mathrm{C}=\mathrm{O})$. Mass $(\mathrm{m} / \mathrm{z}): 546.2(\mathrm{M}+1)$, 503.5 (M-isopropyl), 500.63 (M-NO ${ }_{2}$ ), 369.46, 399.24.

4,4-Difluoro-cyclohexanecarboxylic acid $N^{\prime}$-\{4-hydroxy3,5-dimethoxy-phenyl)methyl]-[3,3-isopropyl-5-methyl)[1,2,4]triazol-4-yl)-8-azabicyclo[3.2.1]oct-8-yl]hydrazide (4j): Dark brown solid, yield: $88.20 \%$, m.p. $130-132{ }^{\circ} \mathrm{C}$. IR $\left(\mathrm{KBr}, v_{\max }, \mathrm{cm}^{-1}\right): 3342,3213,3032,2974,2881,1683,1585$, 1506, 1454, 1421, 1369, 1319, 1276, 1242, 1213, 1163, 1109, 991, 945, 837, 717, 682, 578. ${ }^{1} \mathrm{H}$ NMR (DMSO- $d_{6}, 400 \mathrm{MHz}$ ): $\delta$ 8.665 (s, 2H, -NH-), 8.065 (s, 1H, chiral proton), 7.77-7.78 (s, Ar-H, $1 \mathrm{H}, J=8.69 \mathrm{~Hz}), 6.54-6.56(\mathrm{dd}, 1 \mathrm{H}, J=6.4 \mathrm{~Hz}), 5.1(\mathrm{~s}$, $1 \mathrm{H},-\mathrm{OH}), 3.85\left(\mathrm{~s}, 6 \mathrm{H},-\mathrm{OCH}_{3}\right), 3.73(\mathrm{~m}, 1 \mathrm{H}, J=5.27 \mathrm{~Hz}), 3.23-$ $3.28\left(\mathrm{~m}, 1 \mathrm{H},-\mathrm{CH}\left(\mathrm{CH}_{3}\right)_{2}\right), 2.74\left(\mathrm{~s}, 3 \mathrm{H},-\mathrm{CH}_{3}\right), 2.35(\mathrm{~m}, 3 \mathrm{H}), 1.597-$ $2.17(\mathrm{~m}, 16 \mathrm{H}), 1.29\left(\mathrm{~d}, 6 \mathrm{H}, 2 \times \mathrm{CH}_{3}, J=6.7776 \mathrm{~Hz}\right) .{ }^{13} \mathrm{C}$ NMR: $\delta$ $8.1\left(\mathrm{CH}_{3}\right), 14.2\left(2 \times \mathrm{CH}_{2}\right), 28.4\left(2 \times \mathrm{CH}_{2}\right), 26.3(1 \mathrm{CH})$-isopropyl), $31.8(1 \mathrm{CH}), 34.4\left(2 \times \mathrm{CH}_{2}\right), 36.6\left(2 \times \mathrm{CH}_{2}\right), 42(1 \mathrm{CH}-$ difluoro cyclohexyl ring), $52.7(2 \times \mathrm{CH}), 56.3\left(2 \times \mathrm{OCH}_{3}\right), 75.4$ (1CH, adjacent to arom. ring), 108.3 (1C-geminal fluoro carbon), 108.3, 127, 130.9, 149.9 (4C-arom. ring), $163.7(2 \mathrm{C}$, imine $(\mathrm{C}=\mathrm{N})$, $177.9(\mathrm{C}=\mathrm{O})$.Mass $(\mathrm{m} / \mathrm{z}): 577.32(\mathrm{M}+1), 546.31\left(\mathrm{M}-\mathrm{OCH}_{3}\right)$, $430.27,370.24$.

4,4-Difluoro-cyclohexanecarboxylic acid $N^{\prime}$-\{[3,3-isopropyl-5-methyl)[1,2,4]triazol-4-yl)-8-azabicyclo[3.2.1]oct-8-yl]-(4-isopropyl phenyl)methyl]hydrazide (4k): White solid, yield: $78 \%$, m.p. $118-120^{\circ} \mathrm{C}$. IR $\left(\mathrm{KBr}, v_{\max }, \mathrm{cm}^{-1}\right)$ : 3344 , 3057, 2978, 2885, 1654, 1606, 1573, 1508, 1367, 1267, 1211 , 1111, 1058, 1035, 1012, 954, 835, 785, 680, 582. ${ }^{1} \mathrm{H}$ NMR (DMSO- $\left.d_{6}, 400 \mathrm{MHz}\right): \delta 8.665$ (s, $\left.2 \mathrm{H}, \mathrm{NH}\right), 8.065(\mathrm{~s}, 1 \mathrm{H}$, chiral proton), 6.54-6.56 (dd, $2 \mathrm{H}, J=6.4 \mathrm{~Hz}), 6.77-6.81(\mathrm{dd}, 2 \mathrm{H}, J$ $=6.4 \mathrm{~Hz}), 3.73(\mathrm{~m}, 1 \mathrm{H}, J=5.27 \mathrm{~Hz}), 3.23-3.28(\mathrm{~m}, 1 \mathrm{H},-\mathrm{CH}-$ $\left.\left(\mathrm{CH}_{3}\right)_{2}\right), 2.74\left(\mathrm{~s}, 3 \mathrm{H},-\mathrm{CH}_{3}\right), 2.35(\mathrm{~m}, 3 \mathrm{H}), 1.597-2.17(\mathrm{~m}, 16 \mathrm{H})$, $1.29\left(\mathrm{~d}, 12 \mathrm{H}, 4 \times \mathrm{CH}_{3}, J=6.77 \mathrm{~Hz}\right) .{ }^{13} \mathrm{C}$ NMR: $\delta 8.1\left(\mathrm{CH}_{3}\right)$, $14.2\left(2 \times \mathrm{CH}_{2}\right), 24.4\left(4 \times \mathrm{CH}_{3}\right), 28.4\left(2 \times \mathrm{CH}_{2}\right), 26.3(1 \mathrm{CH})-$ 
isopropyl), $31.8(1 \mathrm{CH}), 34.4\left(2 \times \mathrm{CH}_{2}\right), 36.6\left(2 \times \mathrm{CH}_{2}\right), 42$ (1CH-difluoro cyclohexyl ring), $52.7(2 \times \mathrm{CH}), 74.8(1 \mathrm{CH}$, adjacent to arom. ring), 108.3 (1C-geminal fluoro carbon), $126,128.7,133.5,147.2$ (4C-arom. ring), $163.7(2 \mathrm{C}$, imine $(\mathrm{C}=\mathrm{N}))$, $177.9(-\mathrm{C}=\mathrm{O})$. Mass $(\mathrm{m} / \mathrm{z}): 543.7(\mathrm{M}+1), 500.63$ (M-isopropyl), 424.27, 396.30 .

4,4-Difluoro-cyclohexanecarboxylic acid $N^{\prime}-\{(2$-chloroquinolin-3-yl)-[3-(3-isopropyl-5-methyl)[1,2,4]triazol-4-yl)8-azabicyclo[3.2.1] oct-8-yl-methyl]\}hydrazide (41): Pale yellow solid, yield: $76.25 \%$, m.p. $240-242^{\circ} \mathrm{C}$. IR ( KBr, $v_{\max }$, $\left.\mathrm{cm}^{-1}\right)$ : 3342, 3116, 3012, 2972, 2872, 1668, 1591, 1514, 1398, 1328, 1265, 1224, 1111, 1051, 950, 904, 831, 798, 781, 754. ${ }^{1} \mathrm{H}$ NMR (DMSO- $d_{6}, 400 \mathrm{MHz}$ ): $\delta 9.54$ (s, 2H, NH) $\mathrm{D}_{2} \mathrm{O}-$-exchangeable protons, $8.52-8.50(\mathrm{~d}, 1 \mathrm{H}$, chiral proton), 8.023-7.959 $\left(\mathrm{dd}, 2 \mathrm{H}, J_{1}=8.03 \mathrm{~Hz}, J_{2}=8.28 \mathrm{~Hz}\right), 7.84-7.87(\mathrm{t}, 1 \mathrm{H}, \mathrm{Ar}-\mathrm{H}, J$ $=7.278 \mathrm{~Hz}), 7.732-7.695(\mathrm{t}, 1 \mathrm{H}, \mathrm{Ar}-\mathrm{H}, J=7.278 \mathrm{~Hz}), 4.136-$ $4.175\left(\mathrm{~m}, 1 \mathrm{H}, \mathrm{H}_{\mathrm{a}}, J=5.27 \mathrm{~Hz}\right), 3.48\left(\mathrm{~s}, 3 \mathrm{H},-\mathrm{CH}_{3}\right), 3.28(\mathrm{~m}, 1 \mathrm{H}$, $J=5.27 \mathrm{~Hz}), 2.353(\mathrm{~m}, 3 \mathrm{H}), 2.1-1.97\left(\mathrm{~m}, 8 \mathrm{H}, 4 \times \mathrm{CH}_{2}\right.$, cyclohexane), $1.868(\mathrm{~m}, 4 \mathrm{H}), 1.446(\mathrm{~m}, 4 \mathrm{H}), 1.25-1.26(\mathrm{~d}, 6 \mathrm{H}, J=$ $\left.6.776 \mathrm{~Hz}, 2 \times \mathrm{CH}_{3}\right) \cdot{ }^{13} \mathrm{C}$ NMR: $\delta 8.1\left(\mathrm{CH}_{3}\right), 14.2\left(2 \times \mathrm{CH}_{2}\right), 24.4$ $\left(2 \times \mathrm{CH}_{3}\right), 28.4\left(2 \times \mathrm{CH}_{2}\right), 26.3(1 \mathrm{CH})$-isopropyl), $31.8(1 \mathrm{CH})$, $34.4\left(2 \times \mathrm{CH}_{2}\right), 36.6\left(2 \times \mathrm{CH}_{2}\right), 42$ (1CH-difluoro cyclohexyl ring), $52.7(2 \times \mathrm{CH}), 66.1(1 \mathrm{CH}$, adjacent to arom. ring), 108.3 (1C-geminal fluoro carbon), 126, 128.7, 133.5, 147.2 (4C-arom. ring), 126, 126.3, 126.8, 127.6, 129.2, 130.9, 135.9, 146.8, 157.7 (9C-quinoline ring $)), 163.7(2 \mathrm{C}$, imine $(\mathrm{C}=\mathrm{N})), 177.9(-\mathrm{C}=\mathrm{O})$. Mass (m/e): $586.5(\mathrm{M}+1), 588.5(\mathrm{M}+3), 543.2$ (M-isopropyl), 439.23 .

4,4-Difluoro-cyclohexanecarboxylicacid $N^{\prime}$-\{(3-hydroxynaphthlen-2-yl)-[3-(3-isopropyl-5-methyl)[1,2,4]triazol-4yl)-8-azabicyclo[3.2.1]oct-8-yl-methyl]\}hydrazide (4m): Brown solid, yield: $78.56 \%$, m.p. $182-184^{\circ} \mathrm{C}$. IR (KBr, $v_{\max }$, $\left.\mathrm{cm}^{-1}\right): 3448,3342,3219,3055,2951,2868,1658,1620,1575$, 1544, 1516, 1398, 1367, 1311, 1276, 1240, 1207, 1182, 1109, $1035,1010,941,848,813,738,642 .{ }^{1} \mathrm{H}$ NMR (DMSO- $d_{6}, 400$ $\mathrm{MHz}): \delta 8.75(\mathrm{~s}, 2 \mathrm{H}, \mathrm{NH}) \mathrm{D}_{2} \mathrm{O}$-exchangeable protons, 8.52-8.50 (d, 1H, chiral proton), 8.11 (s, $1 \mathrm{H}, \mathrm{Ar}-\mathrm{H}), 8.023-7.959$ (dd, $2 \mathrm{H}$, $\left.J_{1}=8.03 \mathrm{~Hz}, J_{2}=8.28 \mathrm{~Hz}\right), 7.84-7.87(\mathrm{t}, 1 \mathrm{H}, \mathrm{Ar}-\mathrm{H}, J=7.278$ $\mathrm{Hz}$ ), 7.732-7.695 (t, 1H, Ar-H, J=7.27 Hz), 5.0 (s, 1H, -OH), 4.136-4.175 (m, $\left.1 \mathrm{H}, \mathrm{H}_{\mathrm{a}}, J=5.27 \mathrm{~Hz}\right), 3.48\left(\mathrm{~s}, 3 \mathrm{H},-\mathrm{CH}_{3}\right), 3.28$ $(\mathrm{m}, 1 \mathrm{H}, J=5.27 \mathrm{~Hz}), 2.353(\mathrm{~m}, 3 \mathrm{H}), 2.1-1.97\left(\mathrm{~m}, 8 \mathrm{H}, 4 \times \mathrm{CH}_{2}\right.$, cyclohexane), $1.868(\mathrm{~m}, 4 \mathrm{H}), 1.446(\mathrm{~m}, 4 \mathrm{H}), 1.25-1.26(\mathrm{~d}, 6 \mathrm{H}$, $\left.J=6.776 \mathrm{~Hz}, 2 \times \mathrm{CH}_{3}\right) .{ }^{13} \mathrm{C}$ NMR: $\delta 8.1\left(\mathrm{CH}_{3}\right), 14.2\left(2 \times \mathrm{CH}_{2}\right)$, $24.4\left(2 \times \mathrm{CH}_{3}\right), 28.4\left(2 \times \mathrm{CH}_{2}\right), 26.3(1 \mathrm{CH})$-isopropyl $), 31.8$ $(1 \mathrm{CH}), 34.4\left(2 \times \mathrm{CH}_{2}\right), 36.6\left(2 \times \mathrm{CH}_{2}\right), 42$ (1CH-difluoro cyclohexyl ring), $52.7(2 \times \mathrm{CH}), 65(1 \mathrm{CH}$, adjacent to arom. ring), 108.3 (1C-geminal fluoro carbon), 108.6, 123.3, 125.3, 125.8, 126.9, 127.1, 128.5, 128.8, 132.5, 155.2 (10C-arom. ring), 163.7 $(2 \mathrm{C}$, imine $(\mathrm{C}=\mathrm{N})), 177.9(\mathrm{C}=\mathrm{O})$. Mass $(\mathrm{m} / \mathrm{e}): 567.32(\mathrm{M}+1)$, 524.61, 424.28, 420.55 .

\section{RESULTS AND DISCUSSION}

8-Aza-bicyclo[3.2.1] octyl hydrazide derivatives $\mathbf{4 ( a - m )}$ were synthesized by using modified Mannich reaction and characterized by IR, NMR and mass spectral data. In general, IR absorption band of $1^{\circ}$ amine and $2^{\circ}$ amines of N-H absorption bands observed in the region $3460-3130 \mathrm{~cm}^{-1} .1^{\circ}$ amine $\mathrm{N}-\mathrm{H}$ stretching absorption frequency appeared as doublet at
3460 and $3342 \mathrm{~cm}^{-1}$ for asymmetric and symmetric stretching frequencies. For $2^{\circ}$ amine, the absorption band appears as singlet at $3200-3143 \mathrm{~cm}^{-1}$. The peaks at $3080-3030 \mathrm{~cm}^{-1}$ appeared for aromatic $-\mathrm{C}-\mathrm{H}$ stretching. At $2980-2850 \mathrm{~cm}^{-1}$ methyl C-H stretching absorption was observed. The peak at 1691-1659 $\mathrm{cm}^{-1}$ caused primarily by imine $(\mathrm{C}=\mathrm{N})$, it is conjugation with $\mathrm{C}=\mathrm{C}$ in aromatic ring so it shifts to higher absorption frequency. The out of plane $\mathrm{N}-\mathrm{H}$ wagging is responsible for broad band in the region $844-813 \mathrm{~cm}^{-1}$. The bands at $1597.06-1525 \mathrm{~cm}^{-1}$ are due to the absorption frequency of $\mathrm{C}=\mathrm{C}$ and $\mathrm{N}-\mathrm{H}$ bending vibrations and at $1600-1597 \mathrm{~cm}^{-1}$ absorption is due to $\mathrm{C}=\mathrm{S}$. The peaks at 1274 and $1128-1105 \mathrm{~cm}^{-1}$ for C-N and C-O stretching frequencies, respectively. The band at $1354 \mathrm{~cm}^{-1}$ is attributed for N-N absorption band while at $1045 \mathrm{~cm}^{-1}$ for $\mathrm{C}=\mathrm{S}$ and $758.02 \mathrm{~cm}^{-1}$ for $\mathrm{C}-\mathrm{Cl}$ stretching absorption band.

${ }^{1} \mathrm{H}$ NMR spectra of compounds $4(\mathbf{a}-\mathbf{m})$ revealed that the chemical shift of chiral proton deshielding to down field in the region at $\delta 7.5-8.5 \mathrm{ppm}$ as doublet due to the chiral proton attached to aromatic ring and electronegativity of nitrogen atom on both sides. So it deshields to higher $\delta$ value than expected and for $\mathrm{NH}$ protons appeared as singlet in the region $\delta 12.063$ 11.654 ppm., i.e. one $\mathrm{NH}$ proton at $\delta 12.063$, two hydrogens of $\mathrm{NH}_{2}$ at $\delta 11.83$ and for one $\mathrm{NH}$ proton at $\delta 11.654 \mathrm{ppm}$, these protons are $\mathrm{D}_{2} \mathrm{O}$ exchangeable. The two protons of aromatic ring appeared as doublet of doublet at $\delta 7.59 \mathrm{ppm}$, it's $J_{1}=8.03$ $\mathrm{Hz}$ and $J_{2}=8.28 \mathrm{~Hz}$. One aromatic proton appeared as triplet at $\delta 7.84-7.87 \mathrm{ppm}$ and its coupling constant is $J=7.278 \mathrm{~Hz}$. The other peak at $\delta 7.732-7.695$ appeared as triplet and it's coupling constant is $J=7.278 \mathrm{~Hz}$. The axial proton at azabicyclo[3.2.1] octane ring appeared as multiplet at $\delta 4.136-4.175 \mathrm{ppm}$ having coupling constant $J=5.27 \mathrm{~Hz}$. At $3.48 \mathrm{ppm}$, three hydrogen's of methyl group appeared as singlet and at $\delta 3.18$ appeared as multiplet. The peaks at $\delta 2.353$ appeared as multiplet and at $\delta$ 1.868 and 1.446 appeared as multiplet of $4 \times \mathrm{CH}_{2}$ groups of azabicyclic ring. The six protons of $2 \times \mathrm{CH}_{3}$ groups of isopropyl appeared as doublet with lower intensity with coupling constant $J=6.776 \mathrm{~Hz} .{ }^{13} \mathrm{C} \mathrm{NMR}$ spectra of most compounds have characteristic $\mathrm{C}=\mathrm{O}$ signals appeared at around $\delta 158-177.9 \mathrm{ppm}$, $\mathrm{N}=\mathrm{CH} \delta 163.7-164 \mathrm{ppm}$, at $\delta 108.3 \mathrm{ppm} \mathrm{1C}$-geminal fluoro carbon and $\mathrm{C}=\mathrm{S}$ at $\delta 186 \mathrm{ppm}$.

The synthesized compounds $\mathbf{4}(\mathbf{a}-\mathbf{m})$ have been evaluated for their in vitro antimicrobial activities using four bacterial and two fungal strains. Ciprofloxacin and nystatin have been used as standard drugs for bacteria and fungi. It is revealed that $\mathrm{N}$-\{[3-(3-isopropyl-5-methyl-[1,2,4]triazol-4-yl)-8azabicyclo-[3.2.1]oct-8-yl]-substituted phenyl-methyl\}acid hydrazides and carboxythiamides $\mathbf{4}(\mathbf{a}-\mathbf{m})$ showed significant antimicrobial activities (Table-1). The compounds $\mathbf{4 f}, \mathbf{4 h}, \mathbf{4 i}$, $\mathbf{4 j}, \mathbf{4 l}$ and $\mathbf{4 m}$ exhibited high antibacterial activity while compounds $4 \mathbf{a}, \mathbf{4 d}, \mathbf{4 f}, \mathbf{4 g}, 4 \mathrm{~h}$ and $\mathbf{4 i}$ exhibited high antifungal activity.

\section{ACKNOWLEDGEMENTS}

One of the authors, T. Lakshmi Viveka is thankful to Osmania University, Hyderabad, India for providing the research facilites. The authors also acknowledge to Dr. Y. Rambabu, Indian Institute of Chemical Technology, Hyderabad, India for providing the analytical facilities. 


\begin{tabular}{|c|c|c|c|c|c|c|}
\hline \multicolumn{7}{|c|}{ 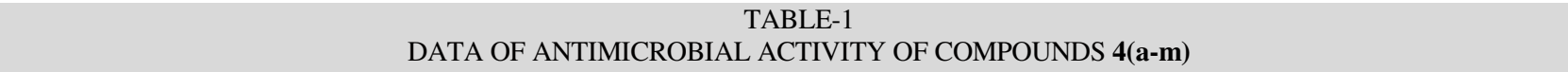 } \\
\hline \multirow{3}{*}{ Compound } & \multicolumn{6}{|c|}{ Zone of inhibition $(\mathrm{mm})$} \\
\hline & \multicolumn{2}{|c|}{ Gram-positive bacteria } & \multicolumn{2}{|c|}{ Gram-negative bacteria } & \multicolumn{2}{|c|}{ Fungi } \\
\hline & $\begin{array}{l}\text { Staphylococcus } \\
\text { aureus }\end{array}$ & $\begin{array}{l}\text { Streptococcus } \\
\quad \text { faecalis }\end{array}$ & $\begin{array}{l}\text { Escherichia } \\
\text { coli }\end{array}$ & $\begin{array}{c}\text { Psedomonas } \\
\text { aeruginosa }\end{array}$ & $\begin{array}{l}\text { Candida } \\
\text { albicans }\end{array}$ & $\begin{array}{l}\text { Aspergillus } \\
\text { niger }\end{array}$ \\
\hline $4 a$ & 20 & 25 & 22 & 21 & 20 & 23 \\
\hline $4 b$ & 16 & 25 & 21 & 19 & 21 & 19 \\
\hline $4 c$ & 23 & 24 & 24 & 19 & 20 & 18 \\
\hline 4d & 24 & 22 & 23 & 21 & 21 & 22 \\
\hline $4 e$ & 18 & 23 & 19 & 22 & 18 & 19 \\
\hline $4 f$ & 23 & 27 & 25 & 22 & 22 & 23 \\
\hline $4 g$ & 20 & 19 & 20 & 24 & 21 & 21 \\
\hline $4 h$ & 27 & 20 & 24 & 25 & 21 & 23 \\
\hline $4 \mathbf{i}$ & 22 & 21 & 25 & 20 & 20 & 21 \\
\hline $4 j$ & 22 & 22 & 21 & 23 & 18 & 20 \\
\hline $4 k$ & 19 & 19 & 20 & 19 & 22 & 19 \\
\hline 41 & 20 & 26 & 20 & 18 & 17 & 23 \\
\hline $4 m$ & 21 & 26 & 24 & 20 & 19 & 22 \\
\hline Standard & 25 & 30 & 28 & 26 & 22 & 24 \\
\hline Std. drug & \multicolumn{4}{|c|}{ Ciprofloxacin (antibacterial) } & \multicolumn{2}{|c|}{ Nystatin (antifungal) } \\
\hline
\end{tabular}

\section{CONFLICT OF INTEREST}

The authors declare that there is no conflict of interests regarding the publication of this article.

\section{REFERENCES}

1. S. Bala, N. Sharma, A. Kajal, S. Kamboj and V. Saini, Int. J. Med. Chem., Article ID 191072 (2014);

https://doi.org/10.1155/2014/191072.

2. H.C. Yao and P. Resnick, J. Org. Chem., 30, 2832 (1965); https://doi.org/10.1021/jo01019a504.

3. R. Fusco and F. Sannicolo, J. Org. Chem., 46, 90 (1981); https://doi.org/10.1021/jo00314a019.

4. T. Iida and F.C. Chang, J. Org. Chem., 46, 2786 (1981); https://doi.org/10.1021/jo00326a037.

5. R.L. Frank and R.R. Phillips, J. Am. Chem. Soc., 71, 2804 (1949); https://doi.org/10.1021/ja01176a060.

6. Y. Koltin, Аnпи. Rep. Med. Chem., 25, 141 (1990).

7. A.J. Abdul Nasser, A. Idhayadhulla, R.S. Kumar and J. Selvin, J. Indian Heterocycl. Chem., 18, 399 (2009).

8. A. Idhayadhulla, R.S. Kumar, A.J. Abdul Nasser, J. Selvin and A. Manilal, Arab. J. Chem., 7, 994 (2014); https://doi.org/10.1016/j.arabjc.2010.12.025.

9. S. Fandakli, H.B. Serapbasoglu, A.D. Meltemyolal and A.K.G. Sengul, Turk. J. Chem., 36, 567 (2012); https://doi.org/10.3906/kim-1103-23.

10. S.G. Subramaniapillai, J. Chem. Sci., 125, 467 (2013); https://doi.org/10.1007/s12039-013-0405-y.

11. T. Plech, M. Wujec, M. Majewska, U. Kosikowska and A. Malm, Med. Chem. Res., 22, 2531 (2013); https://doi.org/10.1007/s00044-012-0248-y.
12. H. Bayrak, A. Demirbas, S.A. Karaoglu and N. Demirbas, Eur. J. Med. Chem., 44, 1057 (2009); https://doi.org/10.1016/j.ejmech.2008.06.019.

13. L. Popiolek, A. Biernasiuk, K. Paruch, P. Patrejko and M. Wujec, Phosphorus, Sulfur, Silicon Rel. Elem., 192, 880 (2017); https://doi.org/10.1080/10426507.2017.1290629.

14. M. Sivakami, B. Natarajan and M. Vijayachandrasekar, Chem. Sci. Transac., 3, 1110 (2014); https://doi.org/10.7598/cst2014.837.

15. M.M. Alam, S. Nazreen, S. Haider, S. Shafi, M.S. Yar, H. Hamid and M.S. Alam, Arch. Pharm., 345, 203 (2012); https://doi.org/10.1002/ardp.201100128.

16. J. Chen, X.Y. Sun, K.Y. Chai, J.S. Lee, M.S. Song and Z.S. Quan, Bioorg. Med. Chem., 15, 6775 (2007); https://doi.org/10.1016/j.bmc.2007.08.004.

17. V. Vrdoljak, I. Dilovic, M. Rubcic, S. Kraljevic Pavelic, M. Kralj, D. Matkovic-Calogovic, I. Piantanida, P. Novak, A. Rozman and M. Cindric, Eur. J. Med. Chem., 45, 38 (2010); https://doi.org/10.1016/j.ejmech.2009.09.021.

18. M. Köksal, N. Gökhan, E. Küpeli, E. Yesilada and H. Erdogan, Arch. Pharm. Res., 30, 419 (2007); https://doi.org/10.1007/BF02980214.

19. D. Sharma, D. Chetia and M. Rudrapal, Asian J. Chem., 28, 782 (2016); https://doi.org/10.14233/ajchem.2016.19478.

20. R. Seth, S. Arora, S. Jain and N. Jain, J. Pharm. Technol. Res. Manage., 3, 57 (2015); https://doi.org/10.15415/iptrm.2015.31005.

21. S.J. Haycock-Lewandowski, A. Wilder and J. Åhman, J. Org. Process. Res. Dev., 12, 1094 (2008); https://doi.org/10.1021/op8000614.

22. T.L. Viveka, M. Saba, S.N.T. Sunitha, Y. Aparna and L.N. Sharada, World J. Pharm. Pharm. Sci., 4, 1087 (2016). 